\title{
Suspected case of subacute stent thrombosis referred for coronary angiography turns out to be unusual aortic dissection presentation
}

\author{
Łukasz Rzepa, Michał Walczewski, Anna Fojt, Robert Kowalik \\ $1^{\text {st }}$ Department of Cardiology, Medical University of Warsaw, Warsaw, Poland
}

Kardiochir Torakochir Pol 2020; 17 (1): 44-46

Acute aortic dissection (AAD) is a life-threatening condition requiring early diagnosis and treatment. Even in quickly diagnosed cases mortality remains high. According to data from the International Registry of Acute Aortic Dissection (IRAD) the in-hospital mortality rate in patients with type A AAD (AAAD) was $22 \%$, and mortality of patients managed surgically was $18 \%$ [1]. AAAD occurs when a tear develops in the ascending part of the aorta and it is a rare cause of chest pain. More frequently patients with chest pain are suspected to suffer from an acute coronary syndrome (ACS). We present a case of a patient with AAAD who was initially mistakenly diagnosed with ACS secondary to stent thrombosis (ST).

A 59-year-old man with history of smoking, hypertension, heart failure in class $I I$ and recent acute myocardial infarction (AMI) was admitted to hospital due to recurrent chest pain. Seven days before admission the patient was discharged from another hospital with diagnosis of anterolateral ST-segment elevation myocardial infarction (STEMI) treated with percutaneous coronary intervention $(\mathrm{PCI})$ with implantation of three stents to the left anterior descending artery and two stents to the right coronary artery. During that hospitalization, transthoracic echocardiography (TTE) showed normal global left ventricular (LV) contractility and ejection fraction of $55 \%$. Additionally, LV hypertrophy and dilatation of the aortic root and the ascending aorta (up to $50 \mathrm{~mm}$ and $48 \mathrm{~mm}$ respectively) were observed. Then he was discharged and he was prescribed dual antiplatelet therapy (DAPT), typical treatment after AMI, and he received referral for a computed tomography (CT) scan of the aorta. However, now, after 7 days, the patient returned to the hospital with recurrent severe chest pain lasting since morning hours and radiating to the shoulders. The patient reported that he had not bought the prescribed medications due to financial problems. The electrocardiogram (ECG), which was different from the discharge ECG, showed new ST-segment elevation (STE) up to $2 \mathrm{~mm}$ and a biphasic $T$ wave in anterior leads. The laboratory test re- vealed a dynamic increase of cardiac troponin (cTn) levels and the patient was transferred to our tertiary care hospital for urgent coronary angiography (CAG). On admission in the cardiac intensive care unit, he reported moderate chest discomfort and intermittent numbness of the right lower limb. His blood pressure (BP) was $110 / 43 \mathrm{~mm} \mathrm{Hg}$ in both arms and his pulse rate was 53 beats/min. On auscultation, murmur above the aortic valve was present. We performed TTE that revealed ascending aorta dissection. It was confirmed in urgent angio-CT scan (Figure 1). The patient underwent an urgent operation during which the cardiopulmonary bypass (CPB) was applied. Resections of the ascending aorta and flaps of the aortic valve were performed, followed by fixation of their prosthetic grafts. Nevertheless, after an attempt of weaning from CPB, the ultra-fragility of vessels caused suture leak and severe bleeding, resulting in drops in BP. Despite achieving hemostasis and massive transfusions, the next attempts of weaning from CPB failed because of low BP. With every minute the procedure became more complicated due to uncontrollable bleeding from surrounding tissue resulting in the patient's death after a 9-hour procedure.

AAD may present with a wide spectrum of signs and symptoms, making it difficult to diagnose in the emergency room (ER). In the case of this patient with recent AMI treated with $\mathrm{PCl}$, the information that he discontinued DAPT directly after discharge strongly indicated ST as a cause of his chest pain. lakovou's study showed that ST occurred in $29 \%$ of patients who prematurely discontinued DAPT after stent implantation, making patients' treatment adherence the most important predictor of ST [2]. The most frequent is subacute ST with onset from $24 \mathrm{~h}$ to 30 days after $\mathrm{PCl}$ and the typical clinical presentation of ST results in symptoms of ACS: typical anginal chest pain and ECG changes usually with STE.

The clinical manifestation of AAD usually clearly differs from that of $A C S$. In the case of $A A D$, the most frequent symptom is a sudden onset of severe chest or back pain [1].

Address for correspondence: Łukasz Rzepa, $1^{\text {st }}$ Department of Cardiology, Medical University of Warsaw, Warsaw, Poland,

e-mail: lukrzepa@gmail.com

Received: 25.07.2019, accepted: 26.12.2019. 

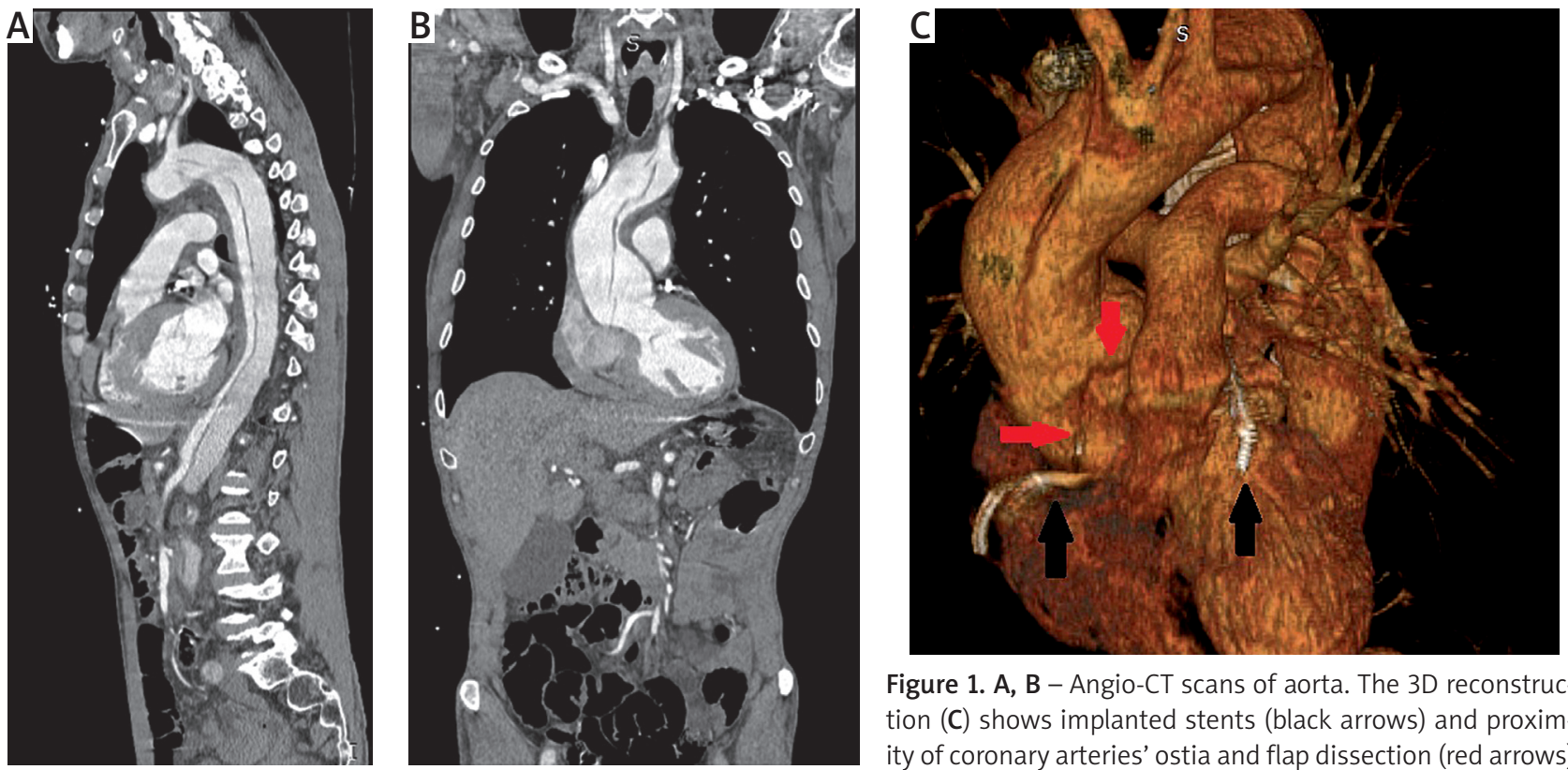

Figure 1. A, B - Angio-CT scans of aorta. The 3D reconstruction (C) shows implanted stents (black arrows) and proximity of coronary arteries' ostia and flap dissection (red arrows)

Chest pain is more common in $A A A D$, whereas back pain is more common in type B AAD. In general, pain in AAD is rather sharp, ripping, tearing and migratory. It is dissimilar to the anginal pain in ST, which is usually described as squeezing, pressure or discomfort in the chest, gradual in onset and offset, lasting longer than 20 minutes and radiating to an upper extremity. However, the pain in AAD can occur in isolation or be associated with syncope, peripheral pulse deficit, heart murmur, hypotension, neurological deficit, or the other clinical symptoms or signs. Therefore, AAD presentation is not always characteristic and may mimic other more common conditions such as ACS including ST, vascular embolization, and abdominal conditions, making its diagnostic process full of pitfalls.

A routine ECG, performed in patients with chest pain, may present typical features of ACS and delay appropriate diagnosis of AAAD. Costin et al. analyzed ECGs of 2,765 patients with AAAD [3]. Among them $60.4 \%$ had different abnormalities in ECG, and only $39.6 \%$ of patients had a normal ECG. Nonspecific ST-T segment changes were noted in $41.8 \%$ of ECGs; $16.2 \%$ of them showed ischemia signs and new $\mathrm{Q}$ waves. STE was observed in $8 \%$ of ECGs. Those findings indicate that abnormalities in ECG in patients with $A A A D$ can either be caused by acute dissection involving coronary arteries ostia, total occlusion by tear of the vessel wall, hematoma compression or can result from chronic underlying diseases such as hypertension or coronary artery disease, existing long before the acute condition [4]. More importantly, in both of those groups cTn levels may be increased. In addition, studies showed that the frequency of cTn elevation is similar in AAAD patients with and without ECG abnormalities [4]. All of these features may imply diagnostic problems of AAAD.

Another very rare pitfall is AAAD presenting as STEMI in $0.5 \%$ of patients. These patients are often directly referred for $C A G$, without awareness of existing AAAD, which finally reveals the correct diagnosis. Luo et al. reported that 11 (5\%) of the 239 patients with AAAD confirmed during hospitalization had initial presentation of AMI (chest pain and elevation of cTn) with a mortality rate of $36 \%$ (4/11). All 6 patients with STE underwent CAG without awareness of AAAD [5]. On the basis of this study the authors suggested that elevated D-dimer with swift TTE performed in the ER before CAG would provide hints of coexistence of AMI and AAAD. However, it is important to underline that routine TTE in each patient with STEMI prior to CAG could delay the time to $\mathrm{PCl}$. Therefore, at first it is recommended to estimate risk of AAD in cases with clinically uncertain diagnosis. The IRAD study showed the high sensitivity (> 95\%) tool for the detection of AAD based on clinical risk markers (high-risk conditions, presenting symptoms, and physical examination findings). Probability of AAD is significantly higher in patients who have one or more risk factors [1]. There are several high risk conditions that greatly increase the likelihood that presenting complaints could be a result of AAD. One of the high risk conditions this patient had was dilatation of the ascending aorta diagnosed in TTE during the first hospitalization. The second one was recent aortic manipulation as $\mathrm{PCl}$. The other high-risk conditions include family history, known aortic valve diseases and Marfan syndrome or other connective tissue diseases, whereas typical for AAD symptoms and physical examination findings included in this tool are those mentioned above that described the character of pain in AAD and associating its symptoms.

In conclusion, in rare cases AAAD presents as ACS and very rarely as STEMI, in combination with history of recent AMI and DAPT discontinuation, making this diagnosis extremely unlikely. For these reasons, this case was tricky but it sends out a clear message that the diagnosis of AAAD requires a high degree of suspicion, the need to perform scrupulous anamnesis, careful physical examination, risk assessment and support of other clinical tools 
such as TTE. This approach would help to avoid misdiagnosis, and reduce delay to proper treatment among patients with AAAD.

\section{Disclosure}

The authors report no conflict of interest.

\section{References}

1. Evangelista A, Isselbacher EM, Bossone E, Gleason TG, Eusanio MD, Sechtem U, Ehrlich MP, Trimarchi S, Braverman AC, Myrmel T, Harris KM, Hutchinson S, O'Gara P, Suzuki T, Nienaber CA, Eagle KA. Insights from the international registry of acute aortic dissection: a 20 -year experience of collaborative clinical research. Circulation 2018; 137: 1846-1860.
2. Iakovou I, Schmidt T, Bonizzoni E, Ge L, Sangiorgi GM, Stankovic G, Airoldi F, Chieffo A, Montorfano M, Carlino M, Michev I, Corvaja N, Briguori C, Gerckens U, Grube E, Colombo A. Incidence, predictors, and outcome of thrombosis after successful implantation of drug-eluting stents. Jama 2005; 293: 2126-2130.

3. Costin NI, Korach A, Loor G, Peterson MD, Desai ND, Trimarchi S, de Vincentiis C, Ota T, Reece TB, Sundt TM, Patel HJ, Chen EP, Montgomery DG, Nienaber CA, Isselbacher EM, Eagle KA, Gleason TG. Patients with type A acute aortic dissection presenting with an abnormal electrocardiogram. Ann Thorac Surg 2018; 105: 92-99.

4. Pourafkari L, Tajlil A, Ghaffari S, Chavoshi M, Kolahdouzan K, Parvizi R, Parizad R, Nader ND. Electrocardiography changes in acute aortic dissection-association with troponin leak, coronary anatomy, and prognosis. Am J Emerg Med 2016; 34: 1431-1436.

5. Luo JL, Wu CK, Lin YH, Kao HL, Lin MS, Ho YL, Chen MF, Chao CL. Type A aortic dissection manifesting as acute myocardial infarction: still a lesson to learn. Acta Cardiol 2009; 64: 499-504. 